\title{
Research on Parallel Design Algorithm of Digital Image
}

\author{
Zhao $\mathrm{Li}^{1, \mathrm{a}}$ \\ ${ }^{1}$ Shandong Yingcai University, Ji'nan, Shandong, 250104, China \\ azhaolifriend@163.com
}

Keywords: Digital image, parallel design, matching, algorithm

\begin{abstract}
In this article, a new method is proposed for the parallel of digital image processing algorithms. The method will transplant parallel the image matching algorithm based on cross correlation to the digital image processing platform.
\end{abstract}

\section{Introduction}

The recently great important and technological problems will rely on the computing technologies' assistance. On the one hand, we will make large scale calculation to get more accurate answer [1,2], on the other hand, computer simulation need to be done in order to further understand the structure and motion law of the problem. The two aspects both rely on parallel processing technology. The area of parallel processing technology is very extensive, including parallel system structure, parallel software, parallel algorithm and so on. The limit of single machine technology determines the computer's development on multi-machine parallel. The development of parallel processing technology will be the main driving force of the future national defense construction and technology development. Therefore, the parallel calculation will be the mainstream in the future. The area of parallel processing technology is very extensive, including parallel system structure, parallel software, parallel algorithm and so on. $[3,4]$

The image plays a very important role in human's perception. We have to contact images any time and any place. The information conveyed by images is more abundant and real than any other means [5]. As an important method of automatization and informatization, the digital image processing technology will be widely used in the various walks of life. Parallel calculation is an effective method to improve the processing speed. And as the development of high performance parallel processing system, the parallel image processing technology provides more space for improving the speed of image processing. Therefore, the research of parallel image processing algorithm is very important and valuable.

In this article, we analyze the problems that parallel algorithm is facing and we propose the corresponding solution. And then we propose a method which can adapt to the digital image processing algorithm. At last, we let an image matching algorithm based on cross correlation transplant into multi-core DSP digital image processing platform. The experimental results show that the method for achieving digital image processing algorithm parallelization method is simple and easy to use. On the basis of high accuracy, it can improve the speed of execution of the image processing algorithm.

\section{The basic theory of parallel algorithm}

It is helpful to understand the effectiveness if the hardware achieves high effect parallel algorithm. According to this principle, we can evaluate those parallel computer system structures. In each aspect (network diameter and the equal width), the optimal network is not present. The two-dimensional network is a kind of network that only keeps the fixed length with the increasing of node number, but it is also the one and only network that has no logarithmic diameter. For example, in the Figure 1 and Figure 2, butterfly and hypercube network have longer equal width. The hypercube network is the only one kind of network that the number of edge is not constant. In the research of parallel computer, there are some "general" computing models. The most influential one is PRAM, BSP and Log P. In addition, there are C3 model and so on. The application 
of these models is very extensive. At first, they provide the designer of parallel algorithm for a basis of design and analysis. If there is a new parallel algorithm, we should have to evaluate the performance. The computation time is expressed by the step number of executing the basic operation. The communication time is got by the calculated communication times and traffic of every step and calculated by computing model (such as Log P, Log GP and so on). Ts represents the serial runtime, Tp represents the parallel runtime. When solving a problem, the total time of all processors is pTp. The Ts part is the time of accomplishing useful work, and the rest is the overhead. Therefore, the overhead function (To) can be expressed:

$$
T_{0}=p T_{p}-T_{s}
$$

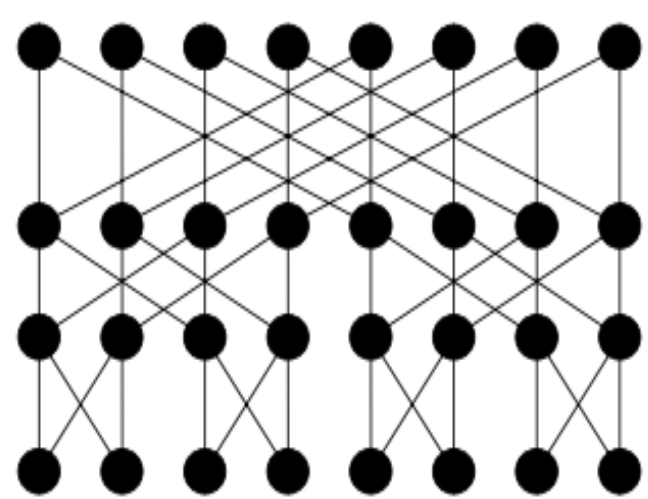

Figure 1. Three-dimensional butterfly structure

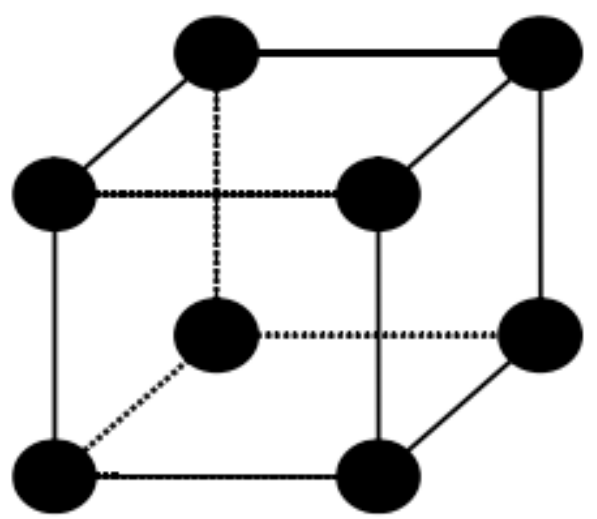

Figure 2. Three-dimensional hypercube structure

The speed-up ratio of the parallel algorithm is defined as:

$$
S_{p}=\frac{T_{s}}{T_{p}}
$$

The efficiency of parallel algorithm is defined as:

$$
E_{p}=\frac{S_{p}}{P}
$$

\section{The design method of parallel digital image processing algorithm}

\section{The distributed arithmetic structure of digital image processing algorithm}

The digital image processing algorithm has obvious distributed arithmetic structure. The Figure 3 shows the basic steps of digital image processing given by Gonzalez.

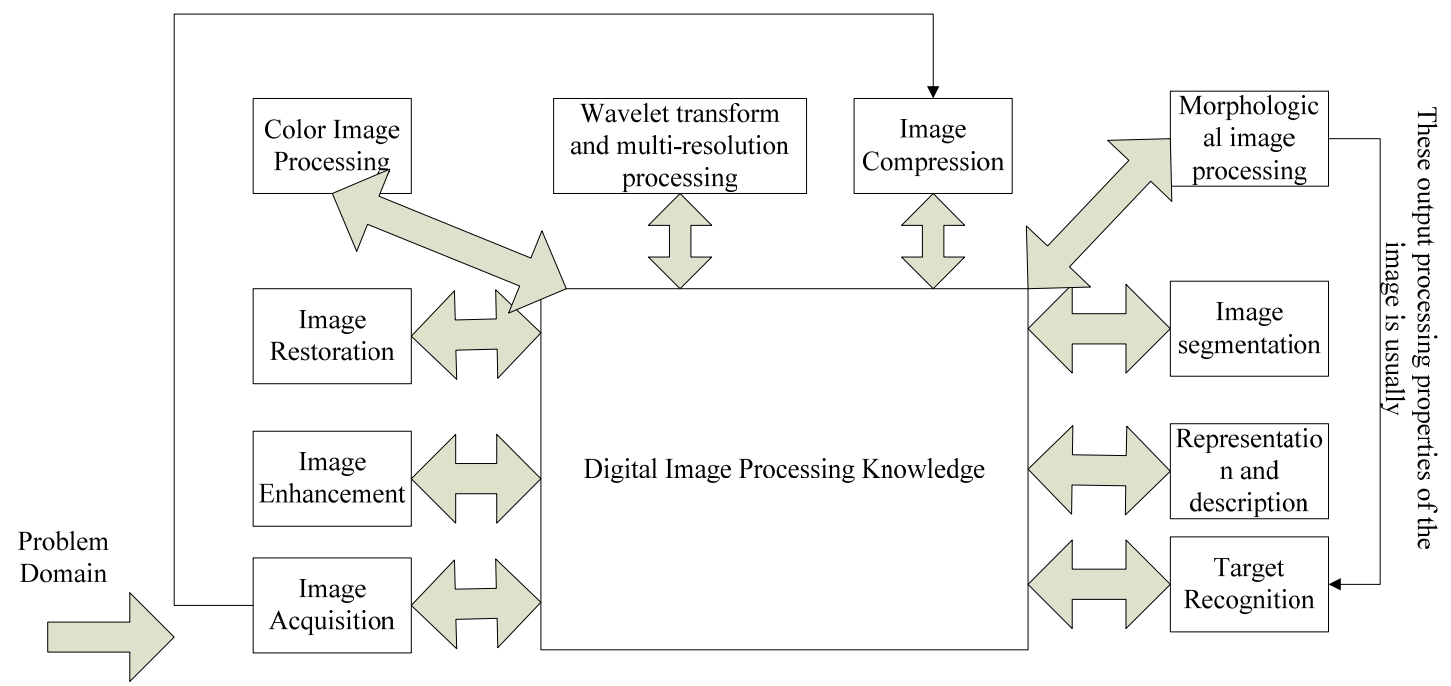

Figure 3. The basic steps of digital image processing

The steps of the image processing can be divided into 3 parts: image preprocessing, data form transformation, the extraction of feature information. Digital images in the computer use a 
continuous space to storage in accordance with the structure of two-dimensional pixel array. Figure 4 shows the storage method of a digital image in a internal storage area.

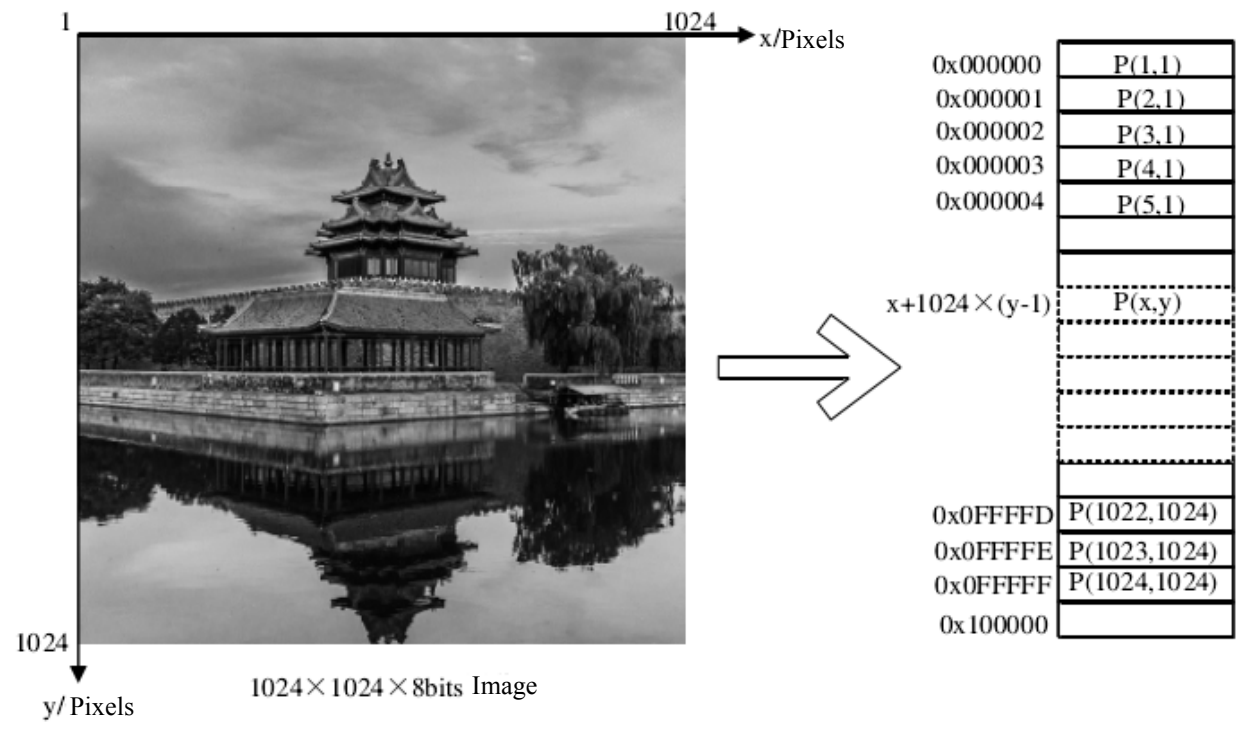

Figure 4. The storage method of digital image in internal storage

\section{The digital image parallel algorithm design}

In the different imaging processes of the same object, images have difference in space and content[5] because of the difference in imaging time, angle, environment and sensor. Image matching is the process of finding the position deviation of unknown image ( real time image or sub-image) in a given image (reference image).

To find a sub-image $w(x, y)$ matching size $J \times K$ in an image $f(x, y)$ of size $M \times N, J \leq M$ and $K \leq N$, so the connection of $f(x, y)$ and $w(x, y)$ can be expressed by the below formula.

$$
c(x, y)=\sum_{s} \sum_{t} f(s, t) w(x+s, y+t)
$$

$x=1,2, \cdots, M-1, y=0,1,2, \cdots N-1$ The occupied area of $c(x, y)$ is the overlap part between $f(x, y)$ and $w(x, y)$.

When the value of $\mathrm{x}$ and $\mathrm{y}$ changes, the w moves in the image. Thus the function $c(x, y)$ is obtained. The C's maximum (W) can match perfectly the location of $f$. In the relative calculation, the error and the $\mathrm{W}$ are proportional.

For a gray image, the value of an arbitrary point $(x, y)$ is the sum of gray values of all the points in the rectangular region constituted by the point of upper left corner to this point:

$$
i(x, y)=\sum_{x} \sum_{y} g(i, j)
$$

The i represents an integral image, $g$ represents the original image. In the practical calculation, the integral image is usually calculated by iterative method. As the formula shows:

$$
i(x, y)=i(x-1, y)+i(x, y-1)-i(x-1, y-1)+g(x, y)
$$

Using the integral image, the average value of all pixels in a rectangular region can be calculated quickly:

$$
\bar{g}=\frac{i\left(x_{1}-y_{1}\right)-i\left(x_{0}-1, y_{1}\right)-i\left(x_{1}, y_{0}-1\right)+i\left(x_{0}-1, y_{0}-1\right)}{\left(x_{1}-x_{0}\right) \times\left(y_{1}-y_{0}\right)}
$$

The cross correlation matching is applied to the two images which have accomplished normalization in the image size, rotation, imitation transformation and so on. That is, cross correlation matching is applied to calculate the plane displacement difference of two images. The computation quantity of cross correlation matching of images is approximately $s \times t$ additive operation and $s \times t$ multiplication operation. However, on the basis of the given integral image, the 
computation quantity of cross correlation matching of images is still approximately $12 \times s \times t$ additive operation plus $5 \times s \times t$ multiplication operation plus $2 \times s \times t$ division operation and one square root operation. In order to reduce the sensitivity of cross correlation to the gray level of the two images, we use the method of the normalization of data instead of the normalization of relative coefficients. The improved algorithm makes the image data after extracting the edge normalized. And it enhances the matching feature of images further by using the morphological erosion algorithm.

The method of using frequency domain to obtain correlation can improve the computation speed. According to properties of 2D Fourier transform, we can obtain:

$$
f(x, y) * h(x, y) \Leftrightarrow F(u, v) H(u, v)
$$

That is, the correlation operation between two images is equivalent to the correlation in the frequency domain. It can use the method of fast correlation to accomplish the cross correlation between the reference image and real time image.

The edge extraction uses Sobel operator. And in the meanwhile, we count the gray total value as the threshold of image binaryzation. The image binaryzation compares each pixel pray value of each 9 neighborhoods with the gray value of the whole image. If the each pixel pray value of each 9 neighborhoods is less than the gray value of the whole image, then the pixel is binaryzated into 0. If the each pixel pray value of each 9 neighborhoods is greater than the gray value of the whole image, then the pixel is binaryzated into 255. The fast 2D Fourier transform(FFT) use the function DSP_fft16x32 to achieve, the function structure is DSP_fft16x32 (const short * restrict ptr_w, int npoints, int * restrict ptr_x, int *restrict ptr_y). The input parameter is rotation factor pointer, the number of transformation, the source data pointer and the result data pointer. The length of input data is $2 k(k=1,2,3 \cdots)$ times than computation points, whose even number is the real part of input data and whose odd number is the imaginary part of the input data. The fast $2 \mathrm{D}$ Fourier transform(FFT) use the function DSP_fft $16 \times 32$ to achieve. The data source of complex multiplication data consist of FFT results of the real-time image and reference image. Each core128 $\mathrm{KB}$ data input space divides into two parts, which respectively receive the FFT results of real-time image and reference image.

The parallel program scheduling model is showed as Figure 5.

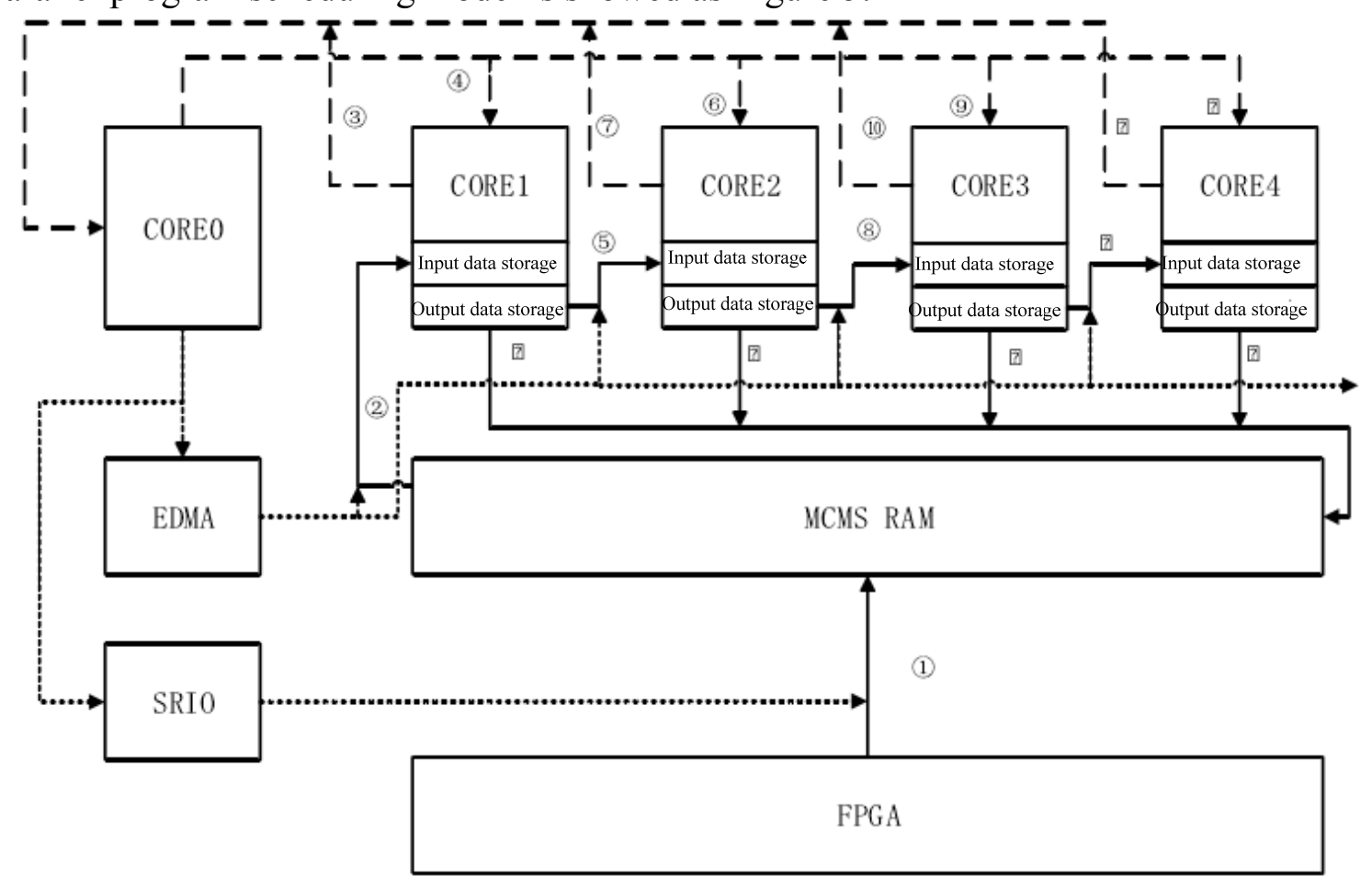

Figure 5. The system action routine of a parallel computing step 
Figure 6 is the basic flow chart of the parallel algorithm:

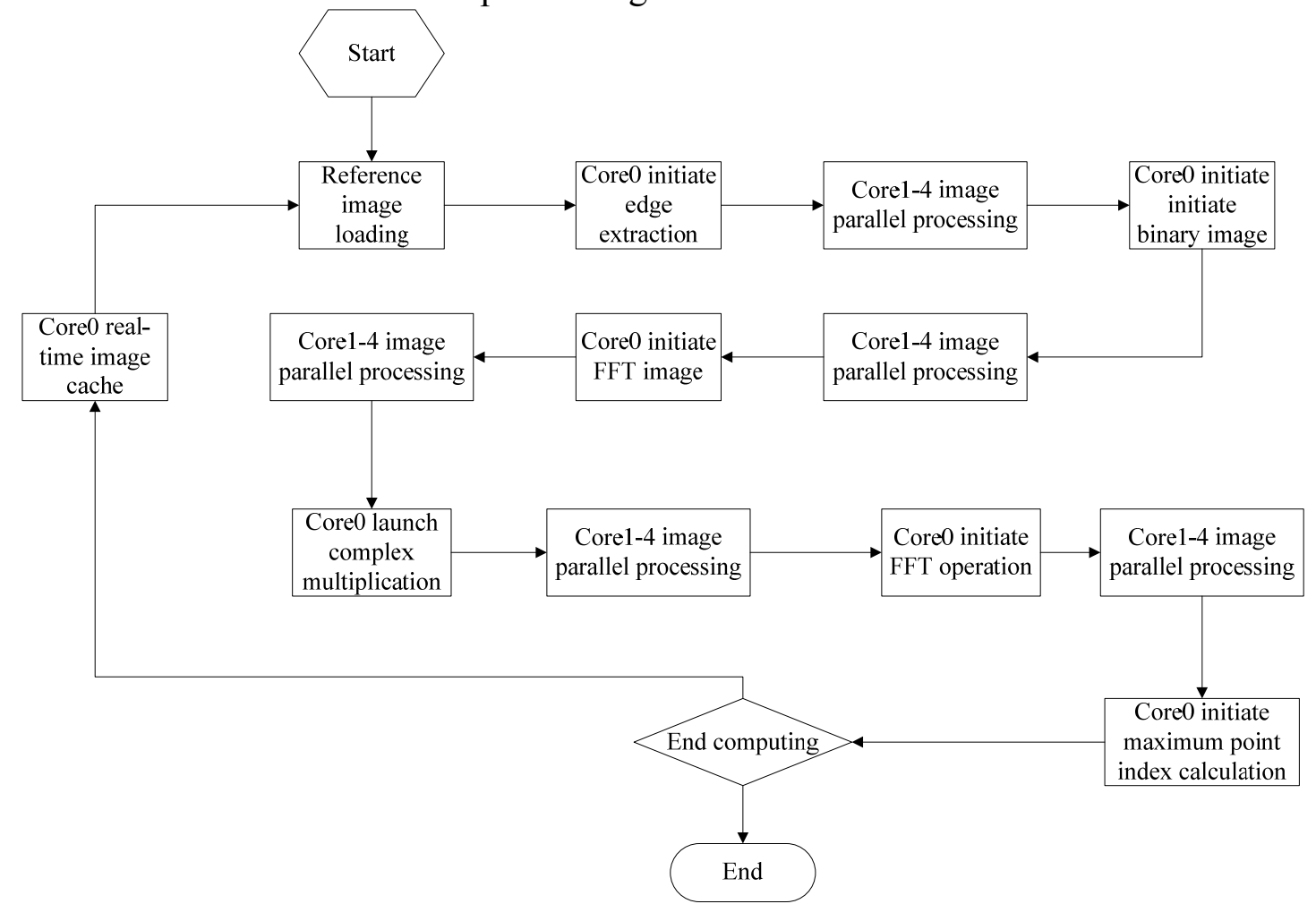

Figure 6. The basic flow chart of the parallel algorithm

The experimental data source uses HD digital images, and it change the position offset of real-time image relative to the reference image by imputing offset. And it can also output the real-time frame rate of digital image. The results of the each step's computation time in parallel algorithm are showed as Table 1. As can be seen from the table, the speed-up ratio of the parallel algorithm is $S=3.4$. After measuring and calculating, it takes up 4400000 CPI because of data communication and scheduling. The proportion of the total time is only about $6 \%$. Thus, the effect of acceleration of core 0 is not obvious. The actual speed-up ratio consists of four parallel operation core. The total operating time has been shortened to less than $100 \mathrm{~ms}$, which meet the design requirements.

Table 1. The number of each step's computation time in parallel algorithm

\begin{tabular}{ccc}
\hline The name of algorithm & $\begin{array}{c}\text { The consumption CPI of } \\
\text { serial algorithm }\end{array}$ & $\begin{array}{c}\text { The consumption CPI of } \\
\text { parallel algorithm }\end{array}$ \\
\hline Edge extraction & $22,214,243$ & $6,533,601$ \\
FFT operation & $80,094,650$ & $23,557,250$ \\
Launch complex & $41,592,329$ & $12,233,038$ \\
multiplication & & \\
Adverse FFT operation & $39,938,712$ & $11,746,680$ \\
Total CPI & $251,691,002$ & $74,025,883$ \\
\hline
\end{tabular}

As the Figure 7 shows, the parallel program is relatively stable in speed-up ratio and efficiency during the execution. With the increasing of data scale, speed-up ratio and efficiency are gradually increasing. With the increasing of data scale, whatever using two nodes or four nodes, the parallel efficiency are gradually increasing. When the data scale is relatively small, the efficiency using two nodes is higher than the efficiency using four nodes. But when the data scale is increasing to a certain degree $(1600 \times 1200)$, the two kinds of efficiency is close to almost coincide in the experimental platform. 


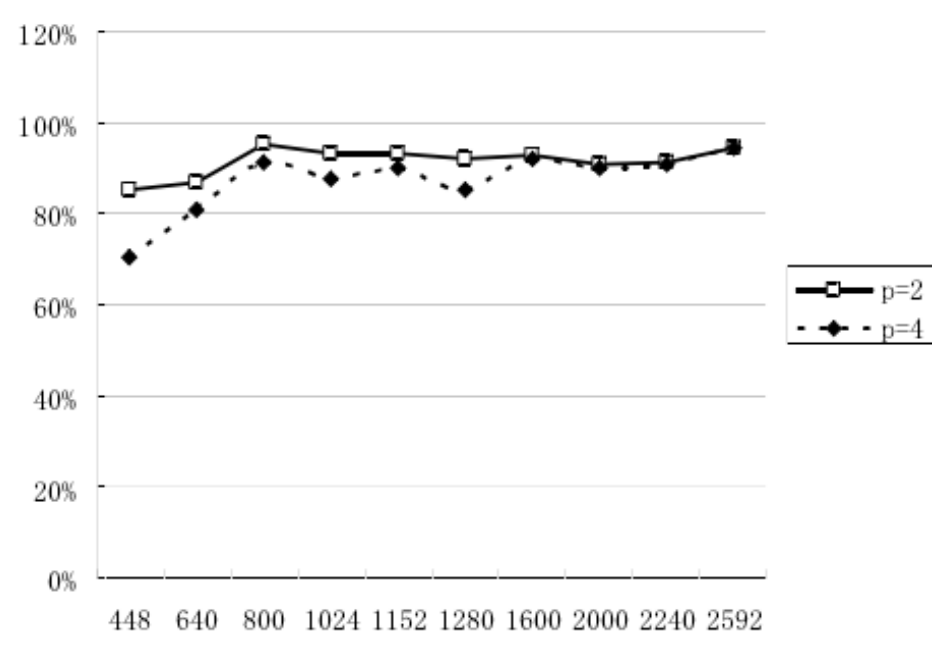

\section{Summary}

Figure 7. The efficiency of parallel algorithm

Through the analysis of the operational characteristics of the basic digital image processing algorithm, we can obtain the distributed computing structure of digital image processing algorithm and the repetitive operation characteristics. And we put forward a design based on parallel digital image processing algorithm and give the performance evaluation of this algorithm. Then we realize a deserialization of cross-correlation matching algorithm by using this design method. The experimental results show that the deserialized cross-correlation image matching algorithm is operating correctly. And after deserialized, the effect of improving the speed of program meet the requirements of the design.

\section{References}

[1] John D. Owens, Mike Houston, David Luebke. 《GPU Computing Proceedings of the IEEE》 2008, Vol. 96, No. 5: 879 899.

[2]WU En-Hua 《State of the Art and Future Challenge on General Purpose Computation by Graphics Processing Unit》 Institute of Software Vol 15(10), 2004, p1493 1504.

[3] Chen Qingqui, Wang Haifeng, Na Lichun 《The research summary of General Purpose Graphics Processing Unit 》 JOURNAL OF NATURAL SCIENCE OF HEILONGJIANG UNIVERSITY Vol 29, 2012,p 672 679..

[4] Wei Zhuoqun 《Parallel Immune Algorithm Based on GPU and Its Application for Production Scheduling of Tandem Cold Mill》 Dalian Dalian University of Technology

[5] Pawan Harish, P.J. 《Narayanan.Accelerating Large Graph Algorithms on the GPU Using CUDA[J]》.HiPC 2007, LNCS 4873, pp. 197-208, $2007 .$.

[6] Alistair Moffat, Andrew Turpin. Compression and Coding Algorithms, Kluwer Academic Publishers, Boston, March 2002.

[7] Wang Shuliang, Ren Lingping, ZhengChengzeng. 《Image compression method based on Wavelet Transform》. COMPUTER ENGINEERING AND APPLICATION Vol 10, 2004, 112-115.

[8] Sun Shixin, Lu Guanghui 《Parallel algorithm and its application》CHINA MACHINE PRESS 